Jpn. J. Limnol., 47, 3, 2299 22:37, 1986.

\title{
A Study on the Structure of the Heterotrophic Bacterial Community in Lake Suwa by Colony Grouping Method
}

\author{
Nobuo Kuroda and Mitsuru Sakamoto
}

\begin{abstract}
The "colony grouping method," a simple method to group many heterotrophic bacterial strains, was applied to analyze the structure of the bacterial community in the natural aquatic environment of Lake Suwa. Among 500 strains obtained from this eutrophic freshwater lake in different months, 150 strains were lost during purification and retransferring procedures. The other 350 strains were exposed to the colony grouping test, and 295 strains were classified into 122. colony groups. The strains included in one colony group showed similar morphological and physiological characteristics. A more detailed description of the structure of the heterotrophic bacterial community in Lake Suwa was accomplished by the colony grouping method rather than the generic grouping method. These indicated that this method would be very useful in the study of the structure of the natural heterotrophic bacterial community. The changes in heterotrophic bacterial flora in terms of colony group in Lake Suwa were suggested to be related to that of algal flora.
\end{abstract}

Key words : bacterial community, colony grouping, classification, Lake Suwa

\section{Introduction}

The heterotrophic bacterial community in natural water is made up of many species having their own specific metabolic characteristics in various substrates. In order to gain a deeper insight into the mechanisms of the cycle of the organic matter in aquatic environments, therefore, the structure of the heterotrophic bacterial community must be revealed in relation to the metabolic characteristics of community members.

There have been several trials to describe the community structure of the heterotrophic bacteria in the aquatic environments by viable countings of bacterial number followed by their isolation and grouping. One of the representative methods is based on taxonomic identification of the isolates, generally at the generic level (SHEWAN et al., 1960; Ishida and Kadota, 1974; Simidu et al., 1977, 1980, 1982; Konda and TkzukA, 1979; Koleshko et al., 1982). Another way to sort bacterial isolates from natural waters is numerical taxonomy (LIGHTHART, 1975; Kaneko et al., 1977; MALlory et al., 1977; Witzel et al., 1982 a, b; MALlory and SAylor, 1983). In these methods, the procedures are generally time -consuming and laborious because of the many isolates and miscellaneous tests to be conducted. Moreover, little information has been provided on the ecological significance of the group classified by these methods (FLKAMI et al., 1981; WITZEL et al., 1982 a).

To overcome these disadvantages, a suitable and simple method must be found to describe the heterotrophic bacterial community at an ecologically significant level. One of the promising procedures for this is the technique proposed by KIUCHI et al. (1968). They grouped isolates from activated sludge by the visible features of a colony formed on different kinds of media, and considered that the isolates classified in one group had identical ecological characteristics. According to Dr. Kuraishi (personal communication), bacterial isolates could be classified by this technique at lower than the generic level. Therefore, this method would be useful to describe the structure of the heterotrophic bacterial community at an ecologically significant level. Furthermore, in the study of the metabolic charac. teristics of the isolates, this method could be used for reduction in the number of strains by selecting one isolate from each classified group.

In the present study, this method, designated here as the "colony grouping method," is applied to describe the structure of the heterotrophic bacterial community in Lake Suwa, and its ecological effectiveness in studying the heterotrophic bacterial community in natural aquatic environments is dis- 
cussed.

\section{Materials and methods}

\section{2-1. Enumeration and isolation of heterotro- phic bacteria.}

The water samples for this study were taken from Lake Suwa. This lake, located in the center of Japan, has a maximum depth of $6.5 \mathrm{~m}$ and a surface area of $1380 \mathrm{ha}$. It is a typical shallow eutrophic lake with a heavy bloom of Microcystis spp. every summer (SAKAMOTo et al., 1975). At its center, surface water samples were manually obtained in a sterilized glass bottle for viable counts and by 51 Van Dorn sampler in plastic bottle for total counts on 10 May, 21 June, 24 July, 18 August and 20 September 1981. As soon as possible after sampling, the water samples were brought to a lakeside laboratory and exposed to treatments for bacterial countings.

Samples for direct counts of total bacteria were fixed with glutaraldehyde (final concentration, $3 \%$ ), and bacterial numbers were determined by the acridine-orange epifluorescent direct count technique after HobBiE et al. (1977) within a few days.

Viable counts of heterotrophic bacteria were made by the pour plate technique. Before dilution, the water samples were well blended with Thermomixer (Thermonics Co., Ltd., Tokyo), in order to make each water sample uniform and disperse aggregated and attached bacteria (Kato and SAKamoto, 1981). For enumeration and isolation of the bacteria and also for stock culture of isolates, the 1/10 PYG medium modified after SAKURAI (1967) was used. It contained $200 \mathrm{mg}$ Trypticase (BBL), $100 \mathrm{mg}$ yeast extract (Difco), $50 \mathrm{mg}$ glucose (Wako) and $12 \mathrm{~g}$ Agar Noble (Difco) in $1,000 \mathrm{ml}$ of GF/C filtered lake water (LW) and was adjusted to $\mathrm{pH} 7.0$. This medium is classified as a dilute medium in organic matter content for bacterial countings in aquatic environments (Yanagita et al., 1978). For isolation and stock culture, LW was diluted twice with distilled water. For stock culture, the soft agar medium $(0.3 \%)$ was used.

After incubation for more than 35 days at $20^{\circ} \mathrm{C}$, the colony number on each plate (2-5 plates per each water sample) was counted with differentiating colony color: white, yellow, orange, pink and other.

Then, colonies with each pigment were picked up (a hundred colonies in total per each water sample) according to the ratio of colony color in the viable counts. After purification by streaking on $1 / 10$
Table 1. Composition of the grouping media.

\begin{tabular}{lcccc}
\hline \multirow{2}{*}{ Content } & & \multicolumn{3}{c}{ Medium } \\
\cline { 3 - 5 } & & B & G & M \\
\hline Polypepton & $(\mathrm{g})$ & 5 & 5 & 5 \\
Beef extract & $(\mathrm{g})$ & 3 & 3 & 3 \\
Yeast extract & $(\mathrm{g})$ & 5 & 3 & 3 \\
Glucose & $(\mathrm{g})$ & - & 5 & - \\
Malt extract & $(\mathrm{g})$ & - & - & 4 \\
Agar & $(\mathrm{g})$ & 12 & 12 & 12 \\
Distilled water & $(\mathrm{ml})$ & 1,000 & 1,000 & 1,000 \\
pH & & 7.0 & 7.0 & 7.0 \\
\hline
\end{tabular}

PYG agar plate repeatedly, each bacterial isolate was kept in soft agar medium for stock culture. These stocked isolates were retransferred to the fresh stock medium every three to four months.

\section{2-2. Grouping of bacterial isolates}

The stocked isolates were grouped by the visible features of their colonies (such as shape, color, surface appearance, transparency, etc.) formed on agar media of different compositions after KIUCH et al. (1968). Instead of the media designed by KiucHi et $a l$., we used newly designed media rich in organic substrates as show in Table 1, since the difference of colony features was more clear than on the original media (Dr. H. Kuraishi, personal communication). It was frequently observed that a bacterial strain formed visually quite different colonies on each of these three media. These three kinds of media (basic, glucose containing and malt extract containing) were respectively designated as medium B, G and $\mathrm{M}$.

After dark incubation on the slant of the grouping media for four weeks at $20^{\circ} \mathrm{C}$, isolates were grouped by comparing the visual features of their colonies with the naked eye. For example, isolates giving visually identical colony features on medium $B$ were classified as one "group on medium B." The same grouping tests were carried out for medium $\mathrm{G}$ and $\mathrm{M}$. and a set of isolates classified into an identical group with any media was determined as one "colony group."

\section{2-3. Generic identification of isolates}

The identity of the stocked isolates was tested at the generic level mainly according to the scheme of Konda and Tezuka (1979), but for Gram-negative chromogenic rods Bergey's Manual of Systematic Bacteriology Vol. 1 (Krieg and HoLT, 1984) was also used for reference. 


\section{Results}

\section{3-1. Enumeration of bacteria}

Viable counts of heterotrophic bacteria and numbers of total bacteria in the surface water of Lake Suwa at five sampling times are shown in Table 2. The viable count values ranged from 2.1 to $13.0 \times 10^{4}$ CFU per $\mathrm{ml}$ and numbers of total bacteria from 2.3 to $10.5 \times 10^{6}$ cells per $\mathrm{ml}$. The ratios of viable count to total count of bacteria in June, July, August and September, 1981 were $0.2 \%, 2.4 \%, 2.4 \%$ and $4.8 \%$, respectively. These values were near the higher limit of the values obtained in many other aquatic environments (DALEY, 1979).

\section{3-2. Colony grouping test}

Among 500 isolates (100 isolates each for five sampling times), 150 isolates were lost during the purification and transferring processes, and the other 350 isolates were exposed to the colony grouping test. Results of the grouping test for final stocked isolates from samples in May, June, July, August and September 1981, are summarized in Table 3.
For the grouping tests, each isolate was preincubated in 1/5 PYG liquid medium. However, in this preincubation, 27 isolates failed to grow. These ungrown isolates were designated as "NL-strain." The other 323 isolates grown in the $1 / 5$ PYG liquid medium were streaked on the agar slopes of the medium B, G and M for further grouping tests.

Twenty-eight isolates which did not form colonies on more than two kinds of the groping media were designated as "NG-strain." Finally, 295 isolates forming colonies on two or three kinds of the media were classified into 122 groups, by comparing their visible colony features (Table 3 ).

The community structure of the heterotrophic bacteria in Lake Suwa is given in Table 4 in terms of the colony group as described above. Out of 122 groups, 84 consisted of a single isolate, and were designated as a "single strain group" (SG). The other 38 groups were made up of two or more isolates. Letters indicating the group (W-, Y-, $\mathrm{P}-$ and $\left.\mathrm{O}^{-}\right)$represent that isolates constituting the group form white, yellow, pink and orange colonies, re-

Table 2. Plate counts of heterotrophic bacteria and direct counts of total bacteria in Lake Suwa.

\begin{tabular}{|c|c|c|c|c|c|}
\hline & 10 May & 21 June & 24 July & 18 August & 20 September \\
\hline Plate count $\left(\times 10^{4} \mathrm{CFU} \mathrm{ml}^{-1}\right)^{*}$ & 7.4 & 2.1 & 11.4 & 13.0 & 11.2 \\
\hline no pigment $\quad \%$ & 24.6 & 50.2 & 48.2 & 38.3 & 29.0 \\
\hline yellow & & 25.1 & 40.4 & 52.8 & 17.6 \\
\hline orange & 75.4 & 246 & 10.5 & 5.9 & 46.5 \\
\hline other pigments (\%) & & 24.6 & 0.9 & 3.1 & 6.6 \\
\hline Direct count $\left(\times 10^{6} \text { cells } \mathrm{ml}^{-1}\right)^{* *}$ & $10.5(0.7)$ & $4.7(1.1)$ & $5.5(1.1)$ & $2.3(0.6)$ & \\
\hline
\end{tabular}

* mean value of counts on two-five plates (CFU : colony forming unit).

Ratio of colonies with different color were also presented for each samples.

${ }^{* *}$ mean value (and standard deviation) of counts on 20 microscope fields.

Table 3. Summary of the colony grouping test I. Number of $\mathrm{NL}^{*}, \mathrm{NG}^{* *}$ and grouped strains and number of the colony group for the each sample.

\begin{tabular}{lrrrrrr}
\hline & May & June & July & \multicolumn{2}{c}{ August September } & Total \\
\hline No. of tested strains & 66 & 74 & 82 & 63 & 65 & 350 \\
No. of NL strains & 6 & - & 2 & 18 & 1 & 27 \\
No. of NG strains & 15 & 3 & 1 & 8 & 1 & 28 \\
No. of grouped strains & 45 & 71 & 79 & 37 & 63 & 295 \\
No. of colony groups & 29 & 42 & 25 & 20 & 20 & 122 \\
\hline
\end{tabular}

*not grown in the liquid medium for preincubation.

** not grown on the two or three kinds of the grouping media. 
spectively.

Four large groups including more than ten isolates (W-5, Y-14, O-2 and O-3) accounted for $29 \%$ of total isolates or $34 \%$ of grouped isolates. Of these large groups, $\mathrm{W}-5, \mathrm{Y}-14$ and $\mathrm{O}-3$ were the dominant groups in July, August and September. The community composition in terms of colony group was simple in July, August and September due to the dominance of these large groups. However, in May and June, the diversity of the composition was high for occurrence of many small (three or less isolates) groups (Table 4).

\section{3-3. Identification of isolates at generic level}

Cell morphology, Gram stain reaction, motility, flagella and a reaction for $\mathrm{O}-\mathrm{F}$ medium were tested to determine the homogeneity of isolates in each colony group. These morphological and physiological characters have been widely used as the principal criteria for the identification of natural bacteria at the generic level (SHEwAN et al., 1960; KONDA and Tezuka, 1979; Oliver, 1982). As shown in Table 5, all the members belonging to one group showed identical characters for these tests. Coincidences in the characters of members belonging to one group were observed in all but two colony groups (Y-14 and $\mathrm{O}^{-1}$ ), in which one isolate had different motility from the others (data not indicated).

Based on the results of these tests, all the isolates including $\mathrm{NG}^{-}$and NL-strains were arranged into 22 generic groups according to the scheme of KONDA and TEZLKA (1979), although catalase and oxidase tests were not carried out. Tentative designations for the generic groups were made as given in Table 6. Since the isolates included in one colony group had the same characters as mentioned above, no colony group spread over two or more generic groups. On the other hand, many generic groups contained two or more colony groups. Such relationships between generic and colony groups are also represented in Table 6 .

The community structure of the heterotrophic bacteria expressed by the generic grouping method is given in Table 7. Pseudomonas (NA) was dominant in June and July, but disappeared in August. Flavobacterium I (yellow and no acid producing type) was the only generic group found through all the months in significant number.

Alcaligenes (Ch) I (yellow pigment producing type) found in four months was dominant in August. Alcaligenes (Ch) II (orange pigment producing type) was remarkably predominating in September.
Table 4. Summary of the colony grouping test II. Seasonal distribution of each colony group.

\begin{tabular}{|c|c|c|c|c|c|c|}
\hline \multirow{2}{*}{$\begin{array}{l}\text { Colony } \\
\text { Group }\end{array}$} & \multicolumn{6}{|c|}{ Number of strains } \\
\hline & May & June & July & August & Septem & Total \\
\hline $\mathrm{W}-1$ & 2 & & & & & 2 \\
\hline $\mathrm{W}-2$ & 3 & & & & & 3 \\
\hline$W-3$ & 2 & 3 & & & & 5 \\
\hline $\mathrm{W}-4$ & 1 & 1 & & & & 2 \\
\hline$W-5$ & 4 & 2 & 29 & & & 35 \\
\hline $\mathrm{W}-6$ & 1 & 8 & & & & 9 \\
\hline $\mathrm{W}-7$ & 1 & 1 & & 1 & & 3 \\
\hline$W-8$ & & 3 & & & & 3 \\
\hline$W-9$ & & 2 & & & & 2 \\
\hline $\mathrm{W}-10$ & & 3 & & & & 3 \\
\hline$W-11$ & & 1 & 3 & & & 4 \\
\hline $\mathrm{W}-12$ & & & 2 & & & 2 \\
\hline$W-13$ & & & & 2 & & 2 \\
\hline $\mathrm{W}-14$ & & & & 2 & 1 & 3 \\
\hline$Y-1$ & 3 & 1 & & & & 4 \\
\hline$Y-2$ & 2 & 1 & & & & 3 \\
\hline$Y-3$ & 3 & & & & & 3 \\
\hline$Y-4$ & 2 & & & & & 2 \\
\hline$Y-5$ & 2 & & & & & 2 \\
\hline$Y-6$ & & 3 & & & & 3 \\
\hline $\mathrm{Y}-7$ & & & 6 & & & 6 \\
\hline $\mathrm{Y}-8$ & & & 4 & & & 4 \\
\hline $\mathrm{Y}-9$ & & & 4 & & & 4 \\
\hline$Y-10$ & & & 2 & & & 2 \\
\hline $\mathrm{Y}-11$ & & & 3 & & & 3 \\
\hline$Y-12$ & & & 2 & & & 2 \\
\hline$Y-13$ & & & 2 & & & 2 \\
\hline $\mathrm{Y}-14$ & & & & 14 & 5 & 19 \\
\hline$Y-15$ & & & & 2 & & 2 \\
\hline $\mathrm{Y}-16$ & & & & 2 & & 2 \\
\hline $\mathrm{Y}-17$ & & & & & 3 & 3 \\
\hline $\mathrm{P}-1$ & 2 & & & & & 2 \\
\hline $\mathrm{P}-2$ & & 5 & & & & 5 \\
\hline $\mathrm{P}-3$ & & 6 & & & & 6 \\
\hline O-1 & & & 4 & & & 4 \\
\hline $\mathrm{O}-2$ & 2 & 4 & 5 & & & 11 \\
\hline $\mathrm{O}-3$ & & & & & 36 & 36 \\
\hline $\mathrm{O}-4$ & & & & & 3 & 3 \\
\hline $\mathrm{SG}^{*}$ & 15 & 27 & 13 & 14 & 15 & 84 \\
\hline
\end{tabular}

* single strain group.

Other generic groups frequently appearing were Moraxella-Acinetobacter, Enterobacteriaceae and Flavobacterium IV (orange and no acid producing type), but they were not predominant in any water samples. 
Table 5. Results of some morphological and physiological tests on the grouped strains. (A part ; Group $\mathrm{W}-1 \sim \mathrm{W}-5$ )

\begin{tabular}{|c|c|c|c|c|c|}
\hline Group & Strain & $\begin{array}{l}\text { Gram } \\
\text { stain }\end{array}$ & $\begin{array}{l}\text { Cell } \\
\text { shape }\end{array}$ & $\begin{array}{l}\text { Motility } \\
\text { Flagella }\end{array}$ & $\mathrm{O}-\mathrm{F}$ test \\
\hline \multirow[t]{2}{*}{ W- 1} & $M-y s-2$ & - & $\mathrm{r}$ & - & $\mathrm{N}$ \\
\hline & M-ys-2 & - & $\mathrm{r}$ & - & $\mathrm{N}$ \\
\hline \multirow[t]{3}{*}{$W-2$} & M-ys-17 & - & $\mathrm{r}$ & + peri & wF \\
\hline & M-ws-3 & - & $\mathrm{r}$ & + peri & $\mathrm{N}-\mathrm{F}$ \\
\hline & $\mathrm{M}-\mathrm{P}-5$ & - & $\mathrm{r}$ & + peri & $\mathrm{O}-\mathrm{wF}$ \\
\hline \multirow[t]{5}{*}{$W-3$} & M-ys-19 & - & $\mathrm{r}$ & + polar & \\
\hline & M-U-2 & - & $r$ & + polar & $\mathrm{N}$ \\
\hline & $\mathrm{Jn}-\mathrm{w}-2$ & - & $\mathrm{r}$ & + polar & $\mathrm{N}$ \\
\hline & $\mathrm{Jn}-\mathrm{w}-12$ & - & $\mathrm{r}$ & + polar & $\mathrm{N}$ \\
\hline & $\mathrm{Jn}-\mathrm{w}-14$ & - & $\mathrm{r}$ & + polar & $\mathrm{N}$ \\
\hline \multirow[t]{2}{*}{$W-4$} & $\mathrm{M}-\mathrm{yco}^{-1}$ & - & $r$ & + peri & $\mathrm{N}$ \\
\hline & $\mathrm{Jn}-\mathrm{w}-33$ & - & $\mathrm{r}$ & + peri & $\mathrm{N}$ \\
\hline \multirow[t]{35}{*}{ W- 5} & M-ws-4 & - & $\mathrm{r}$ & + polar & $\mathrm{N}$ \\
\hline & M-wo-6 & - & $\mathrm{r}$ & + polar & $\mathrm{N}$ \\
\hline & M-wo-7 & - & $\mathrm{r}$ & + polar & $\mathrm{N}$ \\
\hline & M-P-2 & - & $\mathrm{r}$ & + polar & $\mathrm{N}$ \\
\hline & $\mathrm{Jn}-\mathrm{w}-21$ & - & $\mathrm{r}$ & + polar & $\mathrm{N}$ \\
\hline & $\mathrm{Jn}-\mathrm{w}-34$ & - & $\mathrm{r}$ & + polar & $\mathrm{N}$ \\
\hline & $\mathrm{Jl}-\mathrm{w}-3$ & - & $\mathrm{r}$ & + polar & $\mathrm{N}$ \\
\hline & $\mathrm{J} 1-\mathrm{w}-4$ & - & $\mathrm{r}$ & + polar & $\mathrm{N}$ \\
\hline & $\mathrm{Jl}-\mathrm{w}-5$ & - & $\mathrm{r}$ & + polar & \\
\hline & $\mathrm{Jl}-\mathrm{w}-7$ & - & $r$ & + polar & \\
\hline & $\mathrm{J} 1-\mathrm{w}-8$ & - & $\mathrm{r}$ & + polar & $\mathrm{N}$ \\
\hline & $\mathrm{Jl}-\mathrm{w}-9$ & - & $\mathrm{r}$ & + polar & \\
\hline & $\mathrm{Jl}-\mathrm{w}-10$ & - & $\mathrm{r}$ & + polar & \\
\hline & $\mathrm{Jl}-\mathrm{w}-11$ & - & $\mathrm{r}$ & + polar & $\mathrm{N}$ \\
\hline & $\mathrm{Jl}-\mathrm{w}-12$ & - & & + & \\
\hline & $\mathrm{J} 1-\mathrm{w}-13$ & - & $\mathrm{r}$ & + polar & $\mathrm{N}$ \\
\hline & $\mathrm{Jl}-\mathrm{w}-15$ & - & $\mathrm{r}$ & + polar & \\
\hline & $\mathrm{Jl}-\mathrm{W}-16$ & - & $\mathrm{r}$ & + polar & $\mathrm{N}$ \\
\hline & $\mathrm{Jl}-\mathrm{W}-17$ & - & $r$ & + polar & $\mathrm{N}$ \\
\hline & $\mathrm{Jl}-\mathrm{w}-18$ & - & $\mathrm{r}$ & + polar & \\
\hline & J1-W-19 & - & $\mathrm{r}$ & + polar & \\
\hline & $\mathrm{J} 1-\mathrm{w}-22$ & - & $\mathrm{r}$ & + polar & $\mathrm{N}$ \\
\hline & $\mathrm{J} 1-\mathrm{w}-23$ & - & $\mathrm{r}$ & + polar & \\
\hline & $\mathrm{Jl}-\mathrm{w}-24$ & - & $\mathrm{r}$ & + polar & $\mathrm{N}$ \\
\hline & $\mathrm{Jl}-\mathrm{w}-26$ & - & $\mathrm{r}$ & + polar & $\mathrm{N}$ \\
\hline & $\mathrm{Jl}-\mathrm{w}-32$ & - & $\mathrm{r}$ & + polar & \\
\hline & $\mathrm{J} 1-\mathrm{w}-33$ & - & $\mathrm{r}$ & + polar & $\mathrm{N}$ \\
\hline & $\mathrm{Jl}-\mathrm{w}-34$ & - & $\mathrm{r}$ & + polar & $\mathrm{N}$ \\
\hline & $\mathrm{J} 1-\mathrm{W}-35$ & - & $\mathrm{r}$ & + polar & $\mathrm{N}$ \\
\hline & $\mathrm{Jl}-\mathrm{w}-36$ & - & $\mathrm{r}$ & + polar & $\mathrm{N}$ \\
\hline & $\mathrm{J} 1-\mathrm{w}-37$ & - & $\mathrm{r}$ & + polar & $\mathrm{N}$ \\
\hline & $\mathrm{Jl}-\mathrm{w}-38$ & - & $\mathrm{r}$ & + polar & $\mathrm{N}$ \\
\hline & $\mathrm{Jl}-\mathrm{W}-39$ & - & $\mathrm{r}$ & + polar & $\mathrm{N}$ \\
\hline & $\mathrm{Jl}-\mathrm{W}-43$ & - & $\mathrm{r}$ & + polar & \\
\hline & $\mathrm{Jl}-\mathrm{W}-47$ & - & $\mathrm{r}$ & + polar & $\mathrm{N}$ \\
\hline
\end{tabular}

Table 5. continued (Group $\mathrm{Y}-1 \sim \mathrm{Y}-12$ ).

\begin{tabular}{|c|c|c|c|c|c|}
\hline Group & Strain & $\begin{array}{l}\text { Gram } \\
\text { stain }\end{array}$ & $\begin{array}{l}\text { Cell } \\
\text { shape }\end{array}$ & $\begin{array}{l}\text { Motility } \\
\text { Flagella }\end{array}$ & $\mathrm{O}-\mathrm{F}$ test \\
\hline$Y-1$ & $\begin{array}{l}\text { M-ys-10 } \\
\text { M-yco-2 } \\
\text { M-yo-1 } \\
\text { Jn-y-25 }\end{array}$ & $\begin{array}{l}- \\
- \\
- \\
-\end{array}$ & $\begin{array}{l}r \\
r \\
r \\
r\end{array}$ & $\begin{array}{l}+ \text { polar } \\
+ \text { polar } \\
+ \text { polar } \\
+ \text { polar }\end{array}$ & $\begin{array}{l}\mathrm{N} \\
\mathrm{N} \\
\mathrm{N} \\
\mathrm{N}\end{array}$ \\
\hline $\mathrm{Y}-2$ & $\begin{array}{l}\text { M-ys-13 } \\
\text { M-U-1 } \\
\text { Jn-y-4 }\end{array}$ & $\begin{array}{l}- \\
- \\
-\end{array}$ & $\begin{array}{l}r \\
r \\
r\end{array}$ & $\begin{array}{l}+ \text { polar } \\
+ \text { polar } \\
+ \text { polar }\end{array}$ & $\begin{array}{l}\mathrm{N} \\
\mathrm{N} \\
\mathrm{N}\end{array}$ \\
\hline $\mathrm{Y}-3$ & $\begin{array}{l}\text { M-ys-22 } \\
\text { M-yco-5 } \\
\text { M-yco-6 }\end{array}$ & $\begin{array}{l}- \\
- \\
-\end{array}$ & $\begin{array}{l}\mathrm{r} \\
\mathrm{r} \\
\mathrm{r}\end{array}$ & $\begin{array}{l}- \\
- \\
-\end{array}$ & $\begin{array}{l}\mathrm{N} \\
\mathrm{N}\end{array}$ \\
\hline $\mathrm{Y}-4$ & $\begin{array}{l}M-y r-2 \\
M-w^{-}-5\end{array}$ & $\begin{array}{l}+ \\
+\end{array}$ & $\begin{array}{l}\mathrm{c} \\
\mathrm{c}\end{array}$ & - & $\mathrm{N}$ \\
\hline $\mathrm{Y}-5$ & $\begin{array}{l}M-w^{-2} \\
M-P-4\end{array}$ & - & $\begin{array}{l}\mathrm{r} \\
\mathrm{r}\end{array}$ & - & $\begin{array}{l}\mathrm{F} \\
\mathrm{F}\end{array}$ \\
\hline$Y-6$ & $\begin{array}{l}\text { Jn-y-1 } \\
\text { Jn-y-5 } \\
\text { Jn-y-20 }\end{array}$ & $\begin{array}{l}- \\
- \\
-\end{array}$ & $\begin{array}{l}\mathrm{r} \\
\mathrm{r} \\
\mathrm{r}\end{array}$ & $\begin{array}{l}+ \text { polar } \\
+ \text { polar } \\
+ \text { polar }\end{array}$ & $\mathrm{N}$ \\
\hline $\mathrm{Y}-7$ & $\begin{array}{l}\mathrm{J} 1-\mathrm{y}-1 \\
\mathrm{~J} 1-\mathrm{y}-3 \\
\mathrm{~J} 1-\mathrm{y}-8 \\
\mathrm{~J} 1-\mathrm{y}-16 \\
\mathrm{~J} 1-\mathrm{y}-23 \\
\mathrm{~J} 1-\mathrm{y}-24\end{array}$ & $\begin{array}{l}- \\
- \\
- \\
- \\
- \\
-\end{array}$ & $\begin{array}{l}\mathrm{r} \\
\mathrm{r} \\
\mathrm{r} \\
\mathrm{r} \\
\mathrm{r} \\
\mathrm{r}\end{array}$ & $\begin{array}{l}- \\
- \\
- \\
- \\
- \\
-\end{array}$ & $\mathrm{O}$ \\
\hline$Y-8$ & $\begin{array}{l}\mathrm{J} 1-\mathrm{y}-10 \\
\mathrm{~J} 1-\mathrm{y}-26 \\
\mathrm{~J} 1-\mathrm{y}-30 \\
\mathrm{~J} 1-\mathrm{y}-38\end{array}$ & $\begin{array}{l}- \\
- \\
- \\
-\end{array}$ & $\begin{array}{l}r \\
r \\
r \\
r\end{array}$ & $\begin{array}{l}- \\
- \\
- \\
-\end{array}$ & $\mathrm{N}$ \\
\hline$Y-9$ & $\begin{array}{l}J 1-y-12 \\
J 1-y-14 \\
J 1-y-31 \\
J 1-y-33\end{array}$ & $\begin{array}{l}- \\
- \\
- \\
-\end{array}$ & $\begin{array}{l}\mathrm{r} \\
\mathrm{r} \\
\mathrm{r} \\
\mathrm{r}\end{array}$ & $\begin{array}{l}- \\
- \\
- \\
-\end{array}$ & $\mathrm{N}$ \\
\hline $\mathrm{Y}-10$ & $\begin{array}{l}\mathrm{J} 1-\mathrm{y}-17 \\
\mathrm{~J} 1-\mathrm{y}-19\end{array}$ & $\begin{array}{l}- \\
-\end{array}$ & $\begin{array}{l}r \\
r\end{array}$ & $\begin{array}{l}- \\
-\end{array}$ & $\mathrm{N}$ \\
\hline$Y-11$ & $\begin{array}{l}\mathrm{J} 1-\mathrm{y}-22 \\
\mathrm{~J} 1-\mathrm{y}-36 \\
\mathrm{~J} 1-\mathrm{y}-40\end{array}$ & $\begin{array}{l}- \\
- \\
-\end{array}$ & $\begin{array}{l}r \\
r \\
r\end{array}$ & $\begin{array}{l}+ \text { peri } \\
+ \text { peri } \\
+ \text { peri }\end{array}$ & $\begin{array}{l}\mathrm{N} \\
\mathrm{N} \\
\mathrm{N}\end{array}$ \\
\hline Y-12 & $\begin{array}{l}J 1-y-25 \\
J 1-y-39\end{array}$ & $\begin{array}{l}- \\
-\end{array}$ & $\begin{array}{l}\mathrm{r} \\
\mathrm{r}\end{array}$ & $\begin{array}{l}- \\
-\end{array}$ & $\begin{array}{l}\mathrm{N} \\
\mathrm{N}\end{array}$ \\
\hline
\end{tabular}

+ : positive reaction, - : negative reaction, $\mathrm{r}$ : rod, c : coccus, polar : polar flagellated, peri : peritrichous flagellated, $\mathrm{N}$ : no acid reaction, $\mathrm{F}$ : fermentative reaction, $\mathrm{O}$ : oxidative reaction, w : weakly. 
Table 6. Designation of the generic group and its relation to the colony group.

\begin{tabular}{|c|c|c|c|c|c|c|}
\hline Generic group ${ }^{1)}$ & $\begin{array}{l}\text { Gram } \\
\text { stain }\end{array}$ & $\begin{array}{l}\text { Cell }^{2)} \\
\text { shape }\end{array}$ & Pigment $^{3)}$ & $\begin{array}{l}\text { Motility }{ }^{4)} \\
\text { Flagella }\end{array}$ & $\begin{array}{l}\mathrm{O}-\mathrm{F} \\
\text { test }^{5)}\end{array}$ & Colony group ${ }^{6)}$ \\
\hline 1. Moraxella-Acinetobacter & - & $\mathrm{r}$ & - & - & $\mathrm{N}$ & W-1, SG(7), NL(2), NG(2) \\
\hline 2. Acinetobacter & - & $\mathrm{r}$ & - & - & $\mathrm{O}$ & $\mathrm{SG}(1)$ \\
\hline 3. Enterobacteriaceae $(\mathrm{Nm})$ & - & $\mathrm{r}$ & - & - & $w F-F$ & $\mathrm{~W}-11, \mathrm{~W}-14, \mathrm{SG}(1)$ \\
\hline 4. Enterobacteriaceae (M) & - & $\mathrm{r}$ & - & + peri & $w F-F$ & $\mathrm{~W}-2, \mathrm{~W}-7, \mathrm{SG}(4)$ \\
\hline 5. Pseudomonas (NA) & - & $\mathrm{r}$ & - & + polar & $\mathrm{N}$ & $\begin{array}{l}W-3, W-5, W-6, W-8, W-9, W-12 \text {, } \\
\text { SG(12), NL(1), NG(1) }\end{array}$ \\
\hline 6. Pseudomonas $(\mathrm{O})$ & - & $\mathrm{r}$ & - & + polar & $\mathrm{N}$ & $\mathrm{SG}(1)$ \\
\hline 7. Alcaligenes & - & $\mathrm{r}$ & - & + peri & $\mathrm{N}$ & $\mathrm{W}-4, \mathrm{SG}(4)$ \\
\hline 8. Agrobacterium-Alcaligenes & - & $\mathrm{r}$ & - & + peri & $\mathrm{O}$ & $\mathrm{W}-10, \mathrm{SG}(2)$ \\
\hline 9. Alcaligenes (Ch) I & - & $\mathrm{r}$ & $\mathrm{Y}$ & + peri & $\mathrm{N}$ & $\mathrm{Y}-11, \mathrm{Y}-14, \mathrm{Y}-15, \mathrm{Y}-17, \mathrm{SG}(4)$ \\
\hline 10. Alcaligenes (Ch) II & - & r & $\mathrm{O}$ & + peri & $\mathrm{N}$ & $\mathrm{O}-1, \mathrm{O}-3, \mathrm{O}-4, \mathrm{SG}(1)$ \\
\hline 11. Flavobacterium-Cytophaga I & - & r & $\mathrm{Y}$ & - & $\mathrm{N}$ & $\begin{array}{l}\text { Y-3, Y-8, Y-9, Y-10, Y-12, Y-13, } \\
\text { SG(24), NL(17), NG(12) }\end{array}$ \\
\hline 12. Flavobacterium-Cytophaga II & - & $\mathrm{r}$ & $\mathrm{Y}$ & - & 0 & $\mathrm{Y}-7, \mathrm{SG}(1)$ \\
\hline 13. Flavobacterium-Cytophaga III & - & $\mathrm{r}$ & $\mathrm{Y}$ & - & $w F-F$ & $\mathrm{Y}-5, \mathrm{Y}-16, \mathrm{SG}(1)$ \\
\hline 14. Flavobacterium-Cytophaga IV & - & $\mathrm{r}$ & $\mathrm{O}$ & - & $\mathrm{N}$ & O-2, SG(5), NL(2), NG(1) \\
\hline 15. Xanthomonas & - & $\mathrm{r}$ & $\mathrm{Y}$ & + polar & $\mathrm{N}$ & $\mathrm{Y}-1, \mathrm{Y}-2, \mathrm{Y}-6, \mathrm{SG}(2), \mathrm{NG}(2)$ \\
\hline 16. Micrococcus I & + & c & - & - & $\mathrm{N}$ & $\mathrm{W}-13$ \\
\hline 17. Micrococcus II & + & c & $\mathrm{Y}$ & - & $\mathrm{N}$ & $\mathrm{Y}-4$ \\
\hline 18. Staphylococcus & + & c & - & - & $\mathrm{F}$ & $\mathrm{SG}(3)$ \\
\hline 19. Unidentified I & - & $\mathrm{r}$ & $\mathrm{O}$ & + polar & $\mathrm{N}$ & $\mathrm{SG}(1)$ \\
\hline 20. Unidentified II & - & $\mathrm{r}$ & $\mathrm{P}$ & + peri & $\mathrm{N}$ & $\mathrm{NG}(2)$ \\
\hline 21. Unidentified III & - & $\mathrm{cr}$ & $\mathrm{P}$ & - & $\mathrm{N}$ & $\mathrm{P}-1, \mathrm{SG}(1), \mathrm{NL}(1)$ \\
\hline 22. Unidentified IV & - & $\mathrm{cr}$ & $\mathrm{P}$ & $+\underset{\text { polar }}{(\text { sub })}$ & $\mathrm{N}$ & $\mathrm{P}-2, \mathrm{P}-3$ \\
\hline
\end{tabular}

1) $\mathrm{Nm}:$ non motile type, $\mathrm{M}:$ motile type, $\mathrm{NA}:$ no acid producing type,

$\mathrm{O}$ : oxidatively acid producing type, $\mathrm{Ch}:$ chromogenic type.

2) $r:$ rod, $c:$ coccus, $c r:$ coccoidal rod.

3) - : no pigment, $\mathrm{Y}:$ yellow, $\mathrm{O}:$ orange, $\mathrm{P}:$ pink.

4) - : non motile, $+:$ motile, peri : peritrichous.

5) $\mathrm{N}:$ no acid reaction, $\mathrm{O}:$ oxidative reaction, $\mathrm{F}:$ fermentative reaction, $w:$ weakly.

6) Number of strains included are given in parentheses.

\section{Discussion}

The present study showed that the colony grouping was a significant method to describe the community structure of the heterotrophic bacteria in Lake Suwa. As shown in Table 6, many generic groups contained two or more colony groups, but one colony group did not spread over two or more generic groups. This fact indicates that the community structure could be described in more detail by the colony grouping method than by generic level identification.

Figure 1 shows the compositions of colony groups for four large generic groups for each sampling time. In Pseudomonas (NA) which increased in the number of strains from May to July, three colony groups (W $-3, W-5$ and $W-6$ ) were common for May and June, and no dominant colony groups were found in these months. However, W-5 found through three months was predominating (29 strains of 38 ) in July. Although Flavobacterium I was the only generic group found through all the months (10-20\% of total isolates), its colony group compositions were completely different from month to month. In this generic group, it was noted that many $\mathrm{NG}^{-}$strains (13 strains) were contained in the May sample and many NL-strains (16 strains) in the August one. In Alcaligenes (Ch) I appearing in May, July, August and September, Y-14 were dominant in August and September, but in the other two months the colony group compositions were quite different. Alcaligenes (Ch) II, extremely predominant in September, was very simple in colony group composition : It was almont completely composed of $0-3$ (36 of 40 strains). 
Table 7. Distribution of the number of strains among each generic group.

\begin{tabular}{|c|c|c|c|c|c|c|}
\hline \multirow{2}{*}{ Generic group } & \multicolumn{6}{|c|}{ Number of strains } \\
\hline & May & June & July & August & September & Total \\
\hline 1. Moraxella-Acinetobacter & 6 & 2 & 1 & 4 & & 13 \\
\hline 3. Enterobacteriaceae $(\mathrm{Nm})$ & & 2 & 3 & 2 & 1 & 8 \\
\hline 4. Enterobacteriaceae (M) & 4 & 3 & 1 & 2 & & 10 \\
\hline 5. Pseudomonas (NA) & 9 & 22 & 38 & & 1 & 70 \\
\hline 7. Alcaligenes & 1 & 4 & & & 1 & 6 \\
\hline 8. Agrobacterium-Alcaligenes & & 5 & & & & 5 \\
\hline 9. Alcaligenes (Ch) I & 2 & & 3 & 18 & 8 & 31 \\
\hline 10. Alcaligenes (Ch) II & & & 4 & & 40 & 44 \\
\hline 11. Flavobacterium-Cytophaga I & 17 & 8 & 18 & 21 & 6 & 70 \\
\hline 12. Flavobacterium-Cytophaga II & & & 7 & & & 7 \\
\hline 13. Flavobacterium-Cytophaga III & 3 & & & 2 & & 5 \\
\hline 14. Flavobacterium-Cytophaga IV & 2 & 5 & 5 & 5 & 2 & 19 \\
\hline 15. Xanthomonas & 8 & 6 & & & & 14 \\
\hline 16. Micrococcus I & & & & 2 & & 2 \\
\hline 17. Micrococcus II & 2 & & & & & 2 \\
\hline 18. Staphylococcus & 3 & & & & & 3 \\
\hline 21. Unidentified III & 3 & & & 2 & & 5 \\
\hline 22. Unidentified IV & & 11 & & & & 11 \\
\hline Other genera & & 2 & & 2 & 1 & 5 \\
\hline Not tested & 6 & 3 & 6 & 6 & 3 & 24 \\
\hline
\end{tabular}
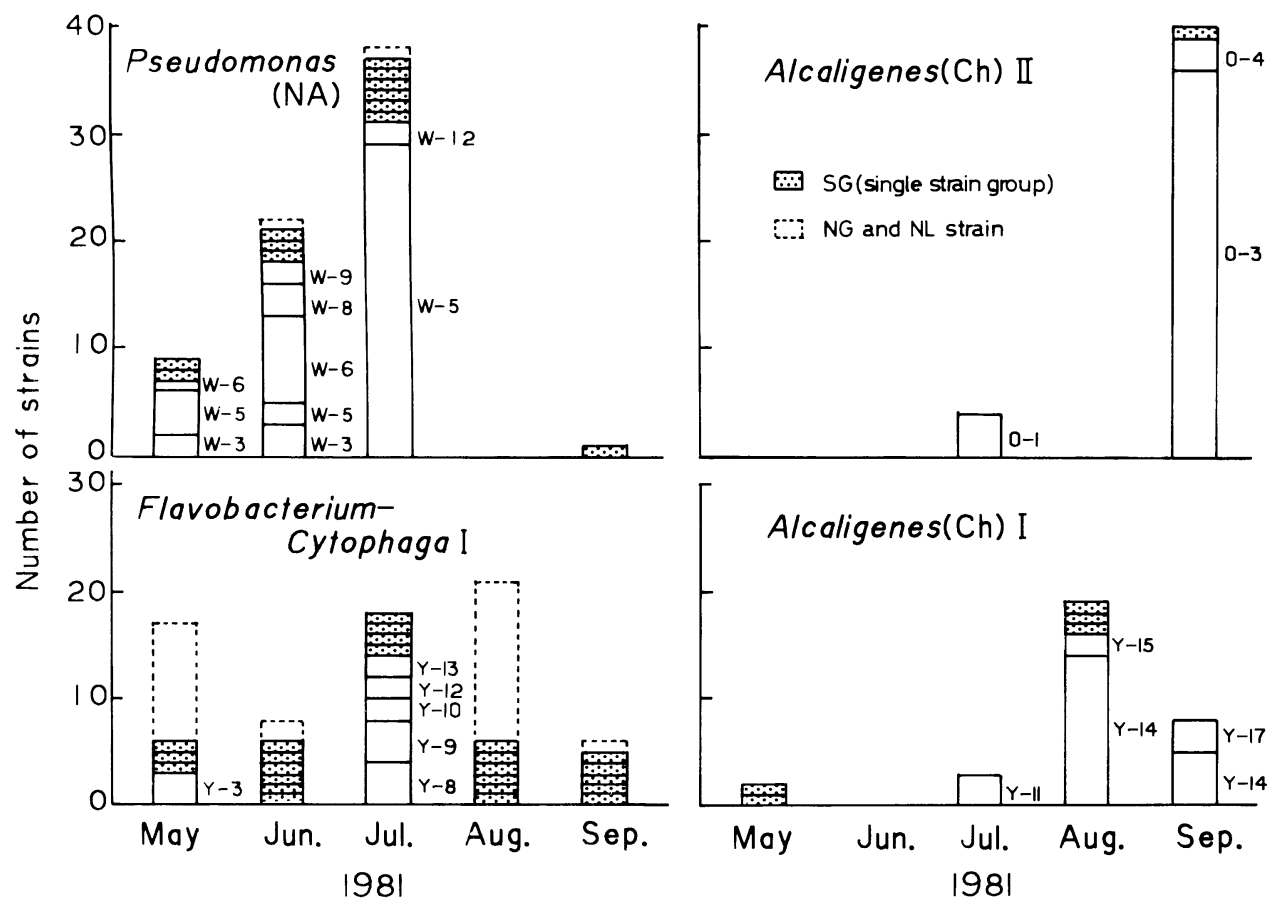

Fig. 1. Distribution of colony groups among the water samples for large generic groups. 
Thus, even in a generic group, the composition of colony groups changes on a monthly basis.

The heterotrophic bacterial floras in Lake Suwa in terms of the colony group (Table 3 and 4) in May and June were of high diversity, while in July, August and September, they were simple by the predominance of one large group. This change in diversity of the group composition seems to be related to that of the phytoplankton flora. In Lake Suwa, a number of algal species (diatoms and green algae) are observed in spring, and a remarkable predominance of Microcystis spp. is found in summer to autumn (SAKAmOTo et al., 1975). In 1981, a high density of Microcystis cells was maintained, with two maxima of biomass, from 20 July to 20 November (Dr. K. Aoy Ama, personal communication). These findings suggest that the changes of bacterial flora might be caused by those of phytoplankton population. The fact that the predominant bacterial groups were different even during the period of the cyanophyta bloom might be related to the production activities of the algal population.

Although "genera" in bacteria is regarded as a transient and often heterogeneous group (COWAN 1955; KRIEG and HolT 1984), a few studies have been made on homogeneity of a genera for isolates from the natural bacterial community. The present study showed that the bacterial community was able to be analysed in more detail by the colony grouping method than by the generic grouping. Moreover, the change of flora in terms of the colony group was well correlated with that of phytoplankton. Therefore, the colony grouping method seems to be a more appropriate method for describing bacterial flora in natural waters.

A preliminary examination, which was undertaken from the physio-ecological viewpoint to examine the homogeneity of the strains belonging to one colony group, also supported our conclusion. Representative strains from several colony groups were cultured in medium with addition of a hot water extract of Microcystis cells collected in Lake Suwa. The strains belonging to one colony group showed the same growth characteristics.

The colony grouping test apparently reduces the laboriousness and time in the study of the structure of the heterotrophic bacterial community. All the isolates belonging to one group proved identical at least at the generic level. We are now pursuing further analyses on the physiological and ecological characteristics of several isolates from each colony group in order to determine the metabolic features of the heterotrophic bacterial community in Lake Suwa.

\section{Acknowledgments}

We wish to express our sincere thanks to Professor Y. SAIJO for his kind encouragement during the course of this study. Thanks are also due to Dr. H. Kuraishi; Tokyo University of Agriculture and Technology, for his valuable advice, and to Mr. $\mathrm{H}$. TERAI, Water Research Institute, Nagoya University, for his useful discussions. We are indebted to Dr. T. OKINO and other members of the Suwa Hydrobiological Station, Shinshu University, for the use of its facilities and their technical assistance in field work, and to Dr. K. Aoyama, Toho University, for his kindness in providing algal data from Lake Suwa.

\section{摘要 \\ コロニーグルーピング法による諏訪湖における 従属栄養細菌群集の構造に関する研究}

1) 自然水域の従属栄養細菌群集の構造を把握, 解 析することを目的とし，細菌株を簡便に識別する万法 である「コロニーグルーピング法」を適用した。

2）諏訪湖湖心表層より，1981 年 5，6，7，8，9月 にそれぞれ 100 株の従属栄養細菌を分離し，保存され た 350 株をコロニーグルーピング試験に供した。

3）前培養で生育した 295 株は，栄養物質組成の異 なる 3 種類の寒天培地に植え, 形成されたコロニーを それぞれの培地ごとに比べることにより，122のグ ループに分けられた。

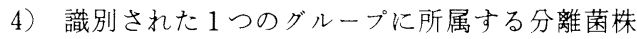
は, 形態学的, 生理学的によく一致した性質をもって いること，本方法によって，従来の同定法を用いた属 レベルでのグループ分けよりも詳しく，従属栄養細菌 群集の構造を把握，解析できること，が示された。

5）コロニーグループを基準にしてみた，諏訪湖従 属栄養細菌群集の変動は, 植物プランクトン群集の変 動と関連していることが示唆された。

\section{References}

Cowan, S. T. (1955) : The phylosophy of classification. J. Gen. Microbiol., 12 : 314-321.

DAley, R. J. (1979) : Direct epifluorescence enumeration of native aquatic bacteria : uses, limitations and comparative accuracy, p. 29-45. In : J. W. Costerton and R. R. Colwell (ed.), Native Aquatic Bacteria: Enumeration, Activity, and Ecology. American Society For Testing And Materials. 
Frkill, K., U.Sinmel and N. TAga (1981): A Microbiological study on the decline process of a phytoplankton bloom in Aburatsubo Inlet, Kanagawa, Japan. Bull. Plank. Soc. Jap., 28 : 33 -41 .

Hobbie, J. E., R. J. Daley, and S. Jasper (1977) : Use of nuclepore filters for counting bacteria by fluorescence microscopy. Appl. Environ. Microbiol., $33: 1225^{-1228 .}$

IsHidA, Y. and H. Kanota (1974) : Ecological studies on bacteria in the sea and lake waters polluted with organic substances-I : responses of bacteria to different concentrations of organic substances. Bull. Jap. Soc. Sci. Fish., 40 : 999-1005.

Kaneko, T., R. M. ATlas and M. Krichevsky (1977) : Diversity of bacterial populations in the Beaufort Sea. Nature, 270 : 596-599.

Kato, K., and M. SAKAnoto (1981): Vertical distribution of free-living and attached heterotrophic bacteria in Lake Kizaki. Jap. J. Limnol., 42 : 154 -159 .

Kilchi, K., H. Kiraishi, H. Mirooka, K. Aida and T. UEMiRA (1968): Floc formation in activated sludge I. Floc-forming activities of representative bacteria isolated from activated sludge. J. Gen. Appl. Microbiol., 14: 387-397.

Koleshio, O. I., Yu. S. Poaenko and L. N. Sharan. GOVICH (1982): Numbers and species composition of heterotrophic bacteria in Lake Naroch. Microbiology, 51: 130-132.

Konda, T. and Y. TezukA (1979) : Bacterial flora in the water and sediment of Lake Motosu-ko, an oligotrophic lake in central Japan. Jap. J. Ecol., 29: 209-220.

Krieg, N. R. and J. G. Hol. (1984) : Bergey's Manual of Systematic Bacteriology Vol. 1. Williams \& Wilkins, Baltimore.

Lighthart, B. (1975) A cluster analysis of some bacteria in the water column of Green Lake, Washington. Can, J. Microbiol., 21 : 392-394.

Mallory, L. M., B. Alistin and R. R. Colwell (1977): Numerical taxonomy and ecology of oligotrophic bacteria isolated from the estuarine environment. Can. J. Microbiol., $23: 733-750$.

Mallory, L. M. and G. S. Sayler (1983) : Heterotrophic bacterial guild structure : relationship to biodegradative populations. Microb. Ecol., 9 : 41 -55 .

Oliver, J. D.. (1982): Taxonomic scheme for the identification of marine bacteria. Deep-Sea Res., $29:$ 795-798.
Sakamoto, M., H. Klrasawa and T. Okino (1975) : Productivity and nutrient metabolism of communities in Lake Suwa, p. 107-147. In : S. MoRI and G. Yamamoto (ed.), JIBP Synthesis, vol. 10; Productivity of Communities in Japanese Inland Waters. University of Tokyo Press.

SAKLRAI, Y. (1967): Some examination on the method for enumerating viable heterotrophic bacteria in water. (in Japanese) J. Jap. Biol. Soc. Water Waste, 2:21-27.

Shewan, J. M., G. HobBs and W. Hodgkiss (1960) : A determinative scheme for the identification of certain genera of Gram-negative bacteria, with special reference to the Pseudomonadaceae. J. Appl. Bacteriol., 23 : 379-390.

Sinidu, U., E. Kaneko and N. TAgA (1977): Microbiological studies of Tokyo Bay. Microb. Ecol., $3: 173-191$.

Simidu, U., N. Taga, R. R. Colwell and J. R. Schwarz (1980): Heterotrophic bacterial flora of the seawater from the Nansei Shoto (Ryukyu Retto) area. Bull. Jap. Soc. Sci. Fish., $46: 505-510$.

Simidu, U., K. Tsukamoto and Y. Akagi (1982) : Heterotrophic bacterial population in Bengal Bay and the South China Sea. Bull. Jap. Soc. Sci. Fish., 48: 425-431.

Yanagita, T., T. Ichikawa, T. Tsuji, Y. Kamata, K. ITO and M. SASAKI (1978) : Two trophic groups of bacteria, oligotrophs and eutrophs: their distributions in fresh and sea water areas in the central northern Japan. J. Gen. Appl. Microbiol., $24: 59-88$.

Witzel, K-P., H. J. Overbeck and K. Moaledj (1982): Microbial communities in Lake Pluss - see - an analysis with numerical taxonomy of isolates. Arch. Hydrobiol., 94: 38-52.

Witzel, K-P., K. Moaledj and H. J. Overbeck (1982): A numerical taxonomic comparison of oligocarbophilic and saprophytic bacteria isolated from Lake Pluss-see. Arch. Hydrobiol., 95 : 507-520.

（著者: 黒田伸郎・圾本 充, 名古屋大学水圈科学研 究所, 干 464 名古屋市千種区不老町; Nobuo KURODA and Mitsuru Sakamoto, Water Research Institute, Nagoya University, Furocho, Chikusaku, Nagoya 464, Japan)

Received : 26 July 1985

Accepted : 15 March 1986 